\title{
The support of constructs in thesaurus tools from a Semantic Web perspective: Framework to assess standard conformance
}

\author{
M. Mercedes Martínez-González \\ University of Valladolid, Spain \\ mercedes@infor.uva.es \\ María-Luisa Alvite-Díez \\ University of Leon, Spain \\ luisa.alvite@unileon.es
}

The final, published version of this article is available online. Please check the final publication record for the latest revisions to this article. [Martínez-González, M. M., \& Alvite-Díez, M.L. (2019). The support of constructs in thesaurus tools from a Semantic Web perspective: Framework to assess standard conformance. Computer Standards \& Interfaces. https://doi.org/10.1016/i.csi.2019.02.003]

\begin{abstract}
Thesauri are conceptual tools useful to achieve semantic interoperability and reusability, which are relevant goals in the Semantic Web. Thesaurus standards establish, among other issues, the constructs that can appear in a thesaurus. The ISO 25964 standard for thesauri, which supersedes ISO 2788, is the evolution of the ISO thesauri standard to a conceptual approach closer to the Semantic Web. However, it appeared when SKOS the W3C Recommendation- was already consolidated as the standard for KOS (Knowledge Organization System) representation in the Semantic Web, including thesauri. The evolution from ISO 2788 to ISO 25964, and the relationships between constructs in ISO 2788/ISO 25964 and SKOS, are studied in this paper. From the analysis of this comparison, a methodological framework, that focuses on the construct support, is proposed to evaluate the conformance quality of thesaurus management tools. Target readers are professionals in charge of thesauri edition. A Semantic Web perspective is taken to characterize the effect that using SKOS to represent thesauri can have on the results of the assessment. A proof of concept for the model's feasibility was performed on two tools and the analysis of the results of this experimental validation is presented. The conclusions highlight the model's suitability for assessing conformance to the standards concerning support for thesaurus constructs.
\end{abstract}

\section{Keywords}

ISO 2788; ISO 25964; Semantic Web; SKOS; software conformance; standard conformance; thesaurus constructs; thesaurus standards; thesaurus tools

\section{Introduction}

Thesauri are structured, controlled vocabularies conceived to facilitate information retrieval. Thesauri add to information systems the ability to offer navigation through a thesaurus' categories or to retrieve documents that have been classified under one of its categories. Moreover, the Semantic Web and linked data have brought about a renewed interest in thesauri as conceptual tools that can be used to improve semantic 
interoperability [1-4]. Their potential as support for concept-based searches, i.e., searches in which the user looks for concepts instead of terms in document content $[1,4,5]$ is a valuable feature in the Semantic Web context. The advanced ability of thesauri to manage synonymy, quasi-synonymy and subsumptions between concepts makes them ideal tools to support these searches. Examples of well-known thesauri are: the Agrovoc Thesaurus ${ }^{1}$, created and maintained by the Food and Agriculture Organization of the United Nations (FAO); EuroVoc ${ }^{2}$, the European Union multilingual thesaurus; the Art and Architecture Thesaurus ${ }^{3}$; the UNESCO Thesaurus ${ }^{4}$; the UKAT Thesaurus ${ }^{5}$; the National Agricultural Library Thesaurus (NALT) ${ }^{6}$; and the GEneral Multilingual Environmental Thesaurus (GEMET) ${ }^{7}$.

Standards are the instruments that collect the agreement of a community about the standardized issue. For thesauri, the ISO standards define what a thesaurus is and what the constructs are that a thesaurus can use in order to be a valid controlled vocabulary. Therefore, tools created to assist thesaurus editors should conform to these rules, either by guaranteeing that they are not transgressed, or by warning their users about possible conflicts. In an ideal scenario, a thesaurus tool in accordance with the standard would guarantee that any thesaurus edited with it has constructs conformant with the standard, e.g., automatically inserting any relationships that can be derived from those inserted by end users, etc. Some of these restrictions can be found in ISO 25964-Part 1 [6] and they are collected and expanded in section 3 of this paper. When tools automatically check this conformance, users are greatly helped in correcting errors committed when creating a thesaurus, so they can be more confident about the work done and, consequently, on the effectiveness of the vocabulary. As with ontologies [7], the interest in assisting the end user with a suitable checking tool increases as the size of the KOS being edited is greater.

In this paper, a methodological tool for selecting thesaurus management tools, by assessing their conformance to ISO standards regarding the support of thesaurus constructs, is presented. Its main public is the thesaurus community, i.e., information management professionals involved in the development, maintenance and edition of thesauri, who need reliable thesaurus tools for these tasks. It is worth noting that it is more oriented towards the proper design of thesaurus management tools and their users' experience, rather than a purely theoretical or logical perspective.

The remainder of this paper is organized as follows. Section 2 presents the background and purpose of the work. Section 3 studies the constructs in ISO 2788 and ISO 25964, their evolution from ISO 2788 to ISO 25964 and the equivalence with SKOS constructs. Section 4 presents the methodological framework, the suite of tests used and some indicators. The indicators are used to measure the conformance of thesaurus software in construct support. In section 5 the proof of concept performed on two selected thesaurus tools is outlined. Section 6 is devoted to the discussion of the effects that the evolution of the ISO standard for thesauri has on the methodological framework. The lessons learned from the proof of concept are also included in this section. Finally, section 7 collects the main conclusions obtained.

\footnotetext{
${ }^{1}$ http://aims.fao.org/vest-registry/vocabularies/agrovoc-multilingual-agricultural-thesaurus

2 https://publications.europa.eu/en/web/eu-vocabularies/th-dataset/-/resource/dataset/eurovoc

3 http://www.getty.edu/research/tools/vocabularies/aat/

4 http://vocabularies.unesco.org/browser/thesaurus/en/

5 http://www.ukat.org.uk/

6 https://agclass.nal.usda.gov/agt.shtml

7 https://www.eionet.europa.eu/gemet/en/themes/
} 


\section{Background and purpose}

The ISO 2788 standard [8] established the constructs a thesaurus can have and the integrity restrictions that must be respected. The standard for monolingual thesauri, originally published in 1974, was revised and reedited in 1986. In addition, the ISO 5964 standard [9] establishes the main procedures for the treatment of several languages in the same knowledge resource, identifying the main problems related to semantic equivalence.

However, the "revival" of thesauri as conceptual tools in Semantic Web applications resulted in the update of the thesaurus ISO standard. Between 2011 and 2013, the International Organization for Standardization (ISO) published ISO 25964 [6,10], the thesaurus standard that supersedes ISO 2788. It is an evolution from the approach of term-based thesauri, present in ISO 2788, to concept-based thesauri [11,12]. With the evolution from ISO 2788 to ISO 25964, the thesaurus community embraces the Semantic Web vision. This concept-based approach is closer to the idea of conceptual searches that underlies semantic information retrieval [5,13]. All of this in an environment of semantic interoperability, in which thesauri are reused in open contexts. Reuse is definitely strengthened when using standards to share thesauri ${ }^{8}[1,11]$.

ISO 25964 appeared after the W3C recommendation for representing KOS in the Semantic Web, SKOS (Simple Knowledge Organization System) [14]. SKOS provides a way to represent knowledge organization systems (KOS) using RDF. SKOS is available as an OWL ontology [15]. SKOS already used the concept-based approach. During the period when ISO 2788 was the available standard for thesauri, the benefits of using W3C standards such as SKOS led to several thesauri being represented with SKOS, including the most important thesauri listed in the first paragraph of this Introduction; a list to which new items are constantly being added [12,16]. Moreover, thesaurus tools were adapted to use RDF/SKOS as the underlying language ${ }^{9}$ to store and share the thesauri their users created and edited. However, SKOS is intended for use in a wider set of KOS, including thesauri, but also folksonomies, glossaries, etc. This has required work on the equivalence between the ISO standards and the $\mathrm{W} 3 \mathrm{C}$ standard (SKOS). The equivalence between thesauri constructs, as defined by ISO 2788, and SKOS constructs was first covered by guidelines provided in the SKOS Primer Guide [17]. The equivalence between ISO 25964 and SKOS is dealt with in [18]. Some mappings are direct, but there are correspondences which are more difficult to make ${ }^{10}$.

Just as the ISO standard for thesauri can be compared with SKOS, thesauri can also be compared with ontologies. Thesauri and ontologies are KOS which can be used to organize knowledge from a community. Even though serious efforts have been made to obtain ontologies from thesauri and other KOS [19], there are relevant differences

\footnotetext{
${ }^{8}$ For example, EuroVoc, the thesaurus maintained by the EU Publications Office, is used in a large number of regional parliaments adapted to the specific needs of each institution related to indexing and retrieval, that is, they spawn variants or customizations of the original thesaurus. Adaptations or variants of the ERIC Thesaurus are created for various educational scenarios, as significant political or geographical differences should be addressed in the vocabulary, while variants from the UNESCO thesaurus, in a wider thematic area, serve as additional examples. These examples illustrate that reusing shared thesauri is indeed a common practice.

${ }^{9}$ The term "language" is used here with the same meaning as in the Introduction of the SKOS Recommendation: "SKOS also provides a lightweight, intuitive language for developing and sharing new knowledge organization systems" [14].

${ }^{10}$ For example, concepts in ISO 25964 are concepts in SKOS, but relations like hasSubGroup/hasSuperGroup in ISO 25964 do not have a direct equivalent in SKOS.
} 
between ontologies and thesauri [20,21]. These differences are enough to justify the existence of a Semantic Web standard, SKOS, specifically designed to represent KOS in the Semantic Web context [3]. One relevant difference, enough to support this argument, is provided by the ISO 25964 standard: ontologies deal with classes, which have instances (individuals), while thesauri deal with concepts ${ }^{11}$. It is not in the interest of this paper to get into the analysis of ontologies and thesauri similarities and differences, for which readers are forwarded to the references mentioned above. However, these differences prove that specific work about thesauri is of interest, especially when some thesauri are well established, consolidated KOS that depend on the consensus of wide communities (see, e.g., UNESCO, EuroVoc, Agrovoc, and the other thesauri mentioned in first paragraph of the Introduction), something difficult to achieve when it refers to agreement on what knowledge is relevant for a given community.

However, the use of ontologies is compatible with maintaining thesauri. For example, the use of legal ontologies, such as the one developed by the Publications Office of the European Union [22], is not in conflict with the use by this institution of long-standing thesauri such as EuroVoc in its information systems. In particular, this thesaurus maintained by the Publications Office of the European Union has been selected to illustrate, with examples, the constructs presented in section 3, as well as for the preparation of the ad hoc thesaurus used in the tests of this work.

This is the context in which we place this study. The aim of this work is to provide a methodological framework for selecting thesaurus tools, by assessing their conformance to ISO standards. To achieve this goal the working methodology was based on the literature review and the comparative study of the standards. The target issue of this paper is construct support, a basic issue to ensure the correctness and quality of any thesaurus.

This work is part of a more generic framework [23], initially designed according to the ISO 2788 standard, which is still supported by many thesaurus tools. Here, however, it is presented with the revision needed to adapt it to ISO 25964. In the following, the methodological tool will be referred to as 'the framework'. The term framework is understood here according to [24,25] as a 'software evaluation method suitable for system comparison'. It includes the methodology, the set of issues to evaluate, the set of data (thesauri in this case) to use in the evaluation, and a set of indicators of the quality of software conformance. A relevant advantage of this framework is that the conformance requirements specified by ISO 25964 are covered by a set of tests (see Section 4.2 and the Appendix), each of which operates as an individual instrument with predefined inputs and possible results. Therefore, they can easily be performed by people in charge of selecting a thesaurus tool, who can organize the execution of the set of tests as they prefer, selecting the ones to perform, the order in which they are performed... The individual results of the texts executed can be added later to obtain indicators about the quality of the tool with respect to its conformance to thesauri

\footnotetext{
${ }^{11}$ Reasoning benefits from this, meaning that all individuals or instances inherit the properties of the classes they belong to. However, thesauri deal with concepts, ideas which are not instantiated, i.e., this type of reasoning cannot be applied. For example, in an ontology, "Earth" is an instance of the "Planet" class and inherits the properties of a planet, while in a thesaurus both, "Earth" and "Planet", are concepts. ISO 25964-2:2011 provides an example: "Earth and planets might be two concepts in a thesaurus, linked by a hierarchical BT/NT relationship. Optionally, the instantial nature of the relationship could be made explicit by using the tags BTI and NTI. In an ontology concerned with celestial mechanics, Planet could be established as a class, and Earth as an individual (since the latter is unique in the domain, whereas there is more than one planet). The two could be linked by a class assertion. The presence of such assertions and axioms allows an individual to 'inherit' all the properties of the classes to which it belongs".
} 
standards. This paper provides an enhanced, in-depth and detailed analysis of the construct support problem and the tests designed to evaluate its support in thesaurus tools, none of which could be included in the generic overview presented in reference [23].

This framework is designed for thesauri. Even though thesauri are conceptual tools for knowledge organization, as ontologies and other KOS, their differences justify specific works for each type. In addition, although thesauri must conform to ISO standards, it is worth analyzing thesaurus issues that are also present in SKOS and those that are specific to thesauri, because SKOS provides more flexibility than thesaurus standards (as a thesaurus is just a type of KOS) and because SKOS is widely used to represent thesauri. This analysis provides the key to point out which tests should be supervised more carefully in a practical evaluation because of the probability of obtaining negative results. Thus, in section 3, the SKOS Recommendation is compared with ISO standards.

The method used to adapt the methodological framework is presented in detail in Section 4.1 in order to enable a better understanding of it as a whole. However, we have chosen to bring forward a section dedicated to this issue (Section 3) to present the revision of the constructs in ISO 2788 and ISO 25964 in detail, and the equivalence with SKOS.

In order to provide a proof of concept for the feasibility of the framework, it was applied to two tools, SKOSEd and PoolParty. These results are included. For this work, in which the new ISO 25964 standard is taken into account, tools already using SKOS were considered more interesting. However, while SKOSEd is, as its name indicates, a tool created to edit and manage SKOS systems, PoolParty is a tool specifically created for thesauri. Some differences will be commented on. A person responsible for selecting a thesaurus tool could consider these differences relevant for the selection. However, this kind of decision depends on the intended use of a software, which cannot be foreseen in a generic framework.

This work also differs from those works that test the quality of KOS represented with SKOS [26]. While, in the said works, the goal is to analyze the quality of the SKOS representation of controlled vocabularies (which include other KOS than thesauri) and to provide suggestions to correct the problems detected, in our methodological framework, the interest focuses on helping thesauri editors to select tools that assist them in thesauri edition.

There are surveys of thesaurus tools [27-29]. In these, such features as the creation and management of thesauri, and features related to software output (the display of thesauri on the screen or printer, for instance), have been touched upon. Besides, there are tools to evaluate ontologies and Semantic Web tools [30-32]. However, they are prior to the newest thesaurus standards and the Semantic Web perspective, or they do not offer a specific method for thesaurus tools. The proposal presented here is original due to its Semantic Web oriented approach and its focus on thesauri. In addition, this proposal covers construct support in detail, taking into account ISO 2788, but also SKOS and ISO 25964.

\section{Thesaurus Constructs: from ISO 2788 to ISO 25964. Equivalence with SKOS}

In this section, the constructs that a thesaurus can have are presented. Both constructs in ISO 2788 and ISO 25964 are covered. The parallelism between both standards, and the equivalence with SKOS constructs, are covered at the same time that constructs are 
introduced. The explanations are illustrated with examples from the EuroVoc thesaurus. In addition, when constructs are new additions in ISO 25964, or they acquire a relevance they did not have in ISO 2788 , this is remarked. The equivalence between thesauri constructs, as defined by ISO 2788, and SKOS constructs was first covered by some guidelines provided in the SKOS Primer Guide [17]. The equivalence between the ISO 25964 thesauri constructs and SKOS is dealt with in [18]. It should be noted that although some mappings are rather direct, other equivalences are more open, i.e., there are various possible mappings.

What is presented in this section is essential knowledge to understand the paper. The equivalence between the constructs of both ISO standards and SKOS is the result of the revision carried out as part of the adaptation methodology of the framework (see section 4.1). However, given its intrinsic relationship to the presentation of the constructs, we have decided to present it in this section, to facilitate the reader's understanding of the equivalences between standards, which will be necessary for understanding how to "translate" an ISO construct into a SKOS construct in SKOS oriented thesaurus tools.

\subsection{Constructs}

Thesauri, Concepts and Terms. A thesaurus is a controlled and structured vocabulary. With ISO 2788, the main constructs of thesauri are Terms: words or phrases that represent an idea. ISO 25964 evolves towards the concept-based approach, i.e., the main constructs of thesauri are Concepts, units of thought. Concepts are represented by terms and the relationships that provide structure to a thesaurus are established between concepts.

When mapping to SKOS, skos:ConceptScheme is used to represent a thesaurus, a structure that compiles and organizes a set of concepts, and each concept is represented with skos:Concept, each of them in turn identified with a URI. Labels can be preferred (skos:prefLabel), alternative (skos:altLabel), or hidden (skos:hiddenLabel).

For example, the concept represented with the PreferredTerm 'social rights' in EuroVoc has several SimpleNonPreferredTerm, which can be used to represent the same idea. The mapping to SKOS is direct: this concept is identified with the URI http://eurovoc.europa.eu/3899, classified as a resource of type skos:Concept, and its labels are referred to with the skos:prefLabel, skos:altLabel properties.

It is worth remarking that the idea of Terms in ISO 25964 matches the SKOS-XL extension [33], where terms are raised to the category of objects that can be related with other objects, something which is not possible for labels. skos-xl:label is used in this case. This has been done in EuroVoc 4.7. However, there are few thesauri represented with SKOS-XL and, which may be more influential in real applications, there are few general purpose thesaurus tools that support SKOS-XL in full.

Domains or Microthesauri and Concept groups. Thesauri have usually been organized in thematic fields or domains ${ }^{12}$, also called fields of knowledge or microthesauri. ISO 25964 introduces a new construct: concept groups. Domains are concept groups. Terms (ISO 2788), or concepts (ISO 25964), are grouped in domains. Even if not common, thesauri can have multiple domain levels, that is, domains which are included in other domains. The first ones are subdomains. For instance, the EuroVoc thesaurus has 21 domains -thematic fields- and 127 subdomains or microthesauri. Thus, the domain 'LAW' is divided into seven microthesauri: 'sources and branches of the

\footnotetext{
12 For the rest of this paper, 'domain' will be used.
} 
law', 'civil law', 'criminal law', 'justice', 'organisation of the legal system', 'international law' and 'rights and freedoms'.

ISO 25964 Concept groups are considered a subclass of skos:Collection in [18]. The SKOS Collections are groups of concepts which do not need to be related by hierarchical relationships [17]. Members of a SKOS Collection may come from different ConceptSchemes, which for the interest of thesaurus management means that a SKOS Collection can be used to represent domains or to group concepts from different thesauri. Although the nesting between domains received a dedicated relationship, hasSubGroup/hasSuperGroup, in ISO 25964, there is no equivalence in SKOS [18].

There was a proposal to extend SKOS to make up for this and other shortages, named ISO-THES [34]. However, its documentation is no longer available and hence, it will not be considered in the rest of this study ${ }^{13}$.

Equivalence relationships. An equivalence relationship is established between two terms in a thesaurus representing the same concept in the same natural language. The equivalence relationships are USE (for the preferred term), and UF (for the nonpreferred term). These relationships, present in ISO 2788, are also found in ISO 25964. However, with concept-based thesauri, these relationships are not as crucial in the definition of a thesaurus as they were with ISO 2788, because the idea that was represented by the group of preferred and non-preferred terms is now represented by the concept construct, to which preferred and non-preferred terms are associated. The equivalent for this in SKOS are skos:prefLabel and skos:altLabel respectively ${ }^{14}$.

Thus, in the same example extracted from EuroVoc, mentioned in the introduction of the constructs, the concept with the descriptor (PreferredTerm) 'social rights' in EuroVoc has two SimpleNonPreferredTerm: 'economic and social rights' and 'social freedom'. The three are linked with the concept they qualify by the skos:prefLabel for 'social rights' and skos:altLabel for 'economic and social rights' and 'social freedom', respectively. The equivalence relationship between preferred and non-preferred terms is shown on the web page in EuroVoc by the abbreviations 'UF' (used for), in front of the SimpleNonPreferredTerm and 'USE', in front of the PreferredTerm.

Hierarchical relationships. These relationships were already available in ISO 2788 and provide the hierarchical structure that organizes a thesaurus. They relate a pair of terms (ISO 2788) or concepts (ISO 25964) of which one has a scope falling completely within the scope of the other. They are broader terms (BT) and narrower terms (NT), which are reciprocal. ISO 25964 maintains these traditional symbols, but they should be understood as "broader concept" and "narrower concept" respectively. For example, in EuroVoc, the concept described as 'social rights' has 13 narrower concepts: 'academic freedom', 'equality before the law', 'equal treatment', etc. These relationships have a direct mapping in SKOS: skos:broader and skos:narrower.

\footnotetext{
13 Based on the W3C Recommendation [14], an extension of SKOS for thesauri according to ISO 25964, called ISOTHES (https://lov.linkeddata.es/dataset/lov/vocabs/iso-thes), was proposed. This reference was used as a guide for the study. However, at the time of writing the paper, its documentation was no longer available for a long period. On the other hand, we have not found in the analyzed tools support for ISO-THES. For both these reasons, the search for stable references, and the empirical verification that the SKOS version that the tools support is the Recommendation, without the ISO-THES extension, has led us to focus our study on fully stabilized versions of the standards and accepted by the thesaurus community (thesauri editors, and thesaurus tool developers).

14 There are two possibilities with SKOS: if SKOS Core is used, which is the simplest way of doing it, their value is a literal; if SKOS-XL is used, the labels are related to concepts by these relationships. With SKOS-XL the properties to be used are xl:prefLabel and xl:altLabel.
} 
A novelty in ISO 25964 is that it introduces the possibility to distinguish between three types of hierarchical relationships: generic (kind of, BTG), partitive (part of, BTP) and instantial (instance of, BTI). This has several effects [35]. In addition, this distinction between the three types of hierarchical relationships, as yet, has no mapping in $\mathrm{SKOS}^{15}$. Indeed, a solution was recently proposed in [36], whose essence is to define extended properties (BTGE, BTPE, BTIE). However, it is still too soon to know if it will become a standard solution. Some considerations about this issue are included in the Analysis (Section 6).

Even if not common, hierarchical relationships can be combined in a thesaurus to obtain polyhierarchical structures. Polyhierarchical structures are defined by ISO 259641:2011 as a "hierarchical arrangement of concepts in a thesaurus or classification scheme in which each concept can have more than one broader concept".

Top Terms and Top Concepts. Top concepts are broader than any other concept in their domain. ISO 2788 hasTopTerm/isTopTermOf properties, or the Top Concepts of ISO 25964, do not have a direct mapping in SKOS [18]. Nevertheless, in SKOS, it is possible to state that a concept is a top concept in a concept scheme by using the skos:topConceptOf property. The concept 'social rights' in EuroVoc is a Top Concept of the '1236 rights and freedoms' microthesaurus. That is, it only has NT concepts. Moreover, this is asserted in an RDF representation with the skos:topConceptOf and skos:hasTopConcept properties.

Associative relationships. An associative relationship relates two preferred terms (ISO 2788), or concepts (ISO 25964), that are not in the same hierarchical structure, but are semantically similar. The symbol used is RT (Related Term) and there is a direct mapping in SKOS by means of the skos:related property. ISO 25964 offers the possibility, which was not available in ISO 2788, to comment on the nature of these relationships, something which brings the thesaurus ISO recommendation closer to the ability of ontologies to model semantics in relationships. Following the same previous example, the concept described as 'social rights' has a related concept (RT), 'Community Charter of the Fundamental Social Rights of Workers'. The skos:related property provides this meaning.

Notes. These serve to clarify the meaning and application of a term, or concept, in relation to other terms or concepts in the same thesaurus. Notes can be divided into scope notes, historical notes, editor notes, usage notes, etc. They are both in ISO 2788 and ISO 25964 without changes. SKOS also supports notes. Thesauri editors can add as many notes as they want, or none. Notes do not change the essence of a thesaurus, so no more space will be devoted to this heading.

Arrays. Arrays are groups of sibling concepts. In [18] it is proposed to represent them with skos:Collection. That is, the SKOS collections can be used to represent domains and arrays. We have not found an example of array in the EuroVoc version available in its website. However, for the aim of providing an example here, an array could be made with the set of sibling concepts 'agricultural vehicle, air-cushion vehicle, camping vehicle, electric vehicle, large vehicle, motor vehicle'. All of them have a common

\footnotetext{
${ }^{15} \mathrm{~A}$ W3C draft, which did not evolve to a W3C Recommendation, raised the possibility of a SKOS Extension to support some common features of KOS, especially thesauri. In particular, it introduced generic, partitive and instantial relationships. A. Miles, D. Brickley, SKOS Extensions Vocabulary Specification, World Wide Web Consortium, 2004. https://www.w3.org/2004/02/skos/extensions/spec/2004-10-18.htmlW3C
} 
parent concept, 'vehicle', and this set could be labelled with the characteristic 'type of vehicle'.

Compound equivalence. ISO 25964 introduces the compound equivalence, which was not in ISO 2788. These are relationships used to represent compound concepts that do not exist in a thesaurus but can be expressed as a combination of two or more simpler concepts. In some cases, the new concept is the union of subconcepts (e.g., in EuroVoc 'fossil fuel' could be considered the union of 'coal', 'natural gas', and 'petroleum'). For these situations, [35] recommends using hierarchical relationships with preference to compound equivalence, that is, introducing the compound concept as one more thesaurus concept, which is related by hierarchical relationships with its components. There is no standard solution yet to represent this with SKOS. For instance, the 'fossil fuel' example has been modeled in EuroVoc as a concept, which is related with three other concepts ('coal', 'natural gas', and 'petroleum') by RT relationships. This is not the solution proposed by [35] (RT relationships have been used in EuroVoc instead of hierarchical relationships), but it is closer to its approach than the compound equivalence of ISO 25964.

However, a last remark is worth noting; structures such as the one considered under this heading, compound equivalence, are rarely found in real thesauri, in which simplicity is a design principle.

\subsection{Evolution in constructs from ISO 2788 to ISO 25964}

The main changes introduced by the new ISO standard that affect the framework for construct support assessment are presented and commented in the following.

1. The basic constructs of a thesaurus are no longer terms, but concepts. With this, ISO 25964 has got closer to SKOS. However, ISO 25964 offers the possibility to add relationships between terms if desired. This introduces complexity into the thesauri and difficulties in the mapping to SKOS [18,37].

2. Preferred terms in ISO 2788 should be associated to concepts in ISO 25964. Non-preferred terms should also be associated to concepts.

3. Domains are represented with concept groups in ISO 25964, and these are themselves represented with skos:Collection [18]. This is a change with respect to ISO 2788, as the mapping of domains to SKOS constructs was not fixed. It now becomes clearer what could be expected in tools using SKOS.

4. Nesting between domains is represented in ISO 25964 with the property hasSuperGroup/hasSubGroup. In ISO 2788, there was no equivalent property. The mapping of these properties in SKOS has not yet been solved.

5. New constructs, e.g., compound equivalence, are included in ISO 25964.

6. BT, NT and RT relationships are in both ISO standards with the same semantics. In ISO 2788, they related preferred terms, while in ISO 25964 they relate concepts.

7. However, ISO 25964 introduces a type in NT/BT relationships with three possible values: kind of, part of, and instance of.

8. Top terms find their equivalent in top concepts.

In short, there are new constructs in ISO 25964 and others have changed with respect to the previous standard. They are: Concepts; Terms; Arrays; types in hierarchical relationships: BTG (Generic), BTP (Partitive), BTI (Instantial); Concept groups, used for domains or microthesauri; and Compound equivalences. The possibility of using compound equivalence, or types of hierarchical relationships, supposes a degree of flexibility in thesauri modeling that complicates the decision of designers of thesaurus 
tools about the manner of translating them to their internal representations, thus opening the range of choices available for representing these constructs with SKOS. This can itself result in a similar situation to the one found when domain nesting was evaluated in the previous version of this framework [23]: it would not be surprising if thesauri tools do not provide support for them.

\section{Methodological framework}

The methodological framework, as has already been pointed out, has its starting point in a previous proposal [23]. Now, however, a review of the framework for construct support is offered. This research takes into account the ISO 25964 thesaurus standard, which was not available when the previous framework was developed. Section 4.1 presents the methodology followed for the adaptation of the framework. In section 4.2, the tests in the construct support suite are shown, while heading 4.3 introduces the thesauri used for the evaluation, and heading 4.4 presents a set of indicators which are proposed to measure the quality of the support offered by thesaurus management tools.

\subsection{Methodology for the adaptation of the evaluation framework from ISO 2788 to ISO 25964}

ISO 25964 was used as a guide for the revision carried out. Other possibilities could have been based on the revision of thesaurus tools. However, as their adaptation to the ISO 25964 standard is still in progress, using the standard as a guide was the only guarantee to obtain a revision in which all changes are included, irrespective of whether they have already been reflected in thesaurus tools. The steps followed for the revision are summarized in Figure 1 and listed in the following:

1. Revision of ISO 25964 and the publications treating the main novelties it includes with respect to ISO 2788.

2. Identifying the set of constructs introduced in ISO 25964 for the first time, or that have changes with respect to the previous standard.

3. Comparison of these items with the set of constructs considered in the framework based on ISO 2788. The result was a set of updated constructs, with the mappings between the older list and the new one.

4. Revision of the ISO-SKOS correspondences using the mappings defined in [18].

5. Analysis of the main changes needed to adapt the framework to ISO 25964.

6. Revision of construct support tests to reflect the conclusions from the previous steps.

7. Introduction of a set of indicators, which can be used to measure the quality of the support provided by the tools assessed.

8. Proof of concept. 


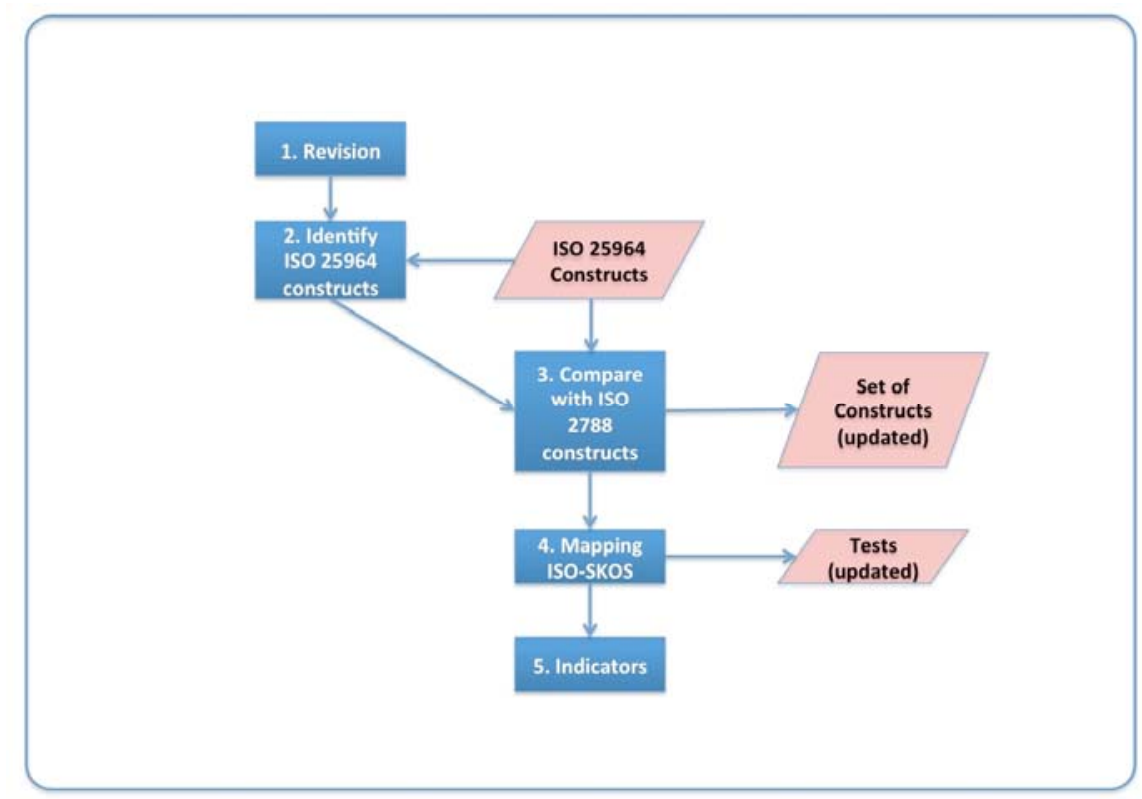

Figure 1. Methodology.

\subsection{Tests to assess the construct support}

This suite of tests is used to check the support of constructs presented in Section 3. The tests are listed in Tables 1 and 2 (column two for ISO 2788 and column three for ISO 25964). Although the construct support suite has been derived from the set of constructs detailed in Section 3, the design of the tests has not always been direct. In this way, it has been decided to include some additional checks when the creation of a given construct should entail the automatic creation of a reciprocal one (e.g., a BT relationship should entail the creation of an equivalent NT relationship). The ability to infer derived relationships could also have been included in the integrity suite, but this would entail repeating the same test twice: once to observe if it supports the construct and another one to observe if it infers the derived relationships. To avoid repetitions, we include it as an aspect to monitor in the execution of the test that checks construct support. Thus, the ability that consists of inferring reciprocal relationships is covered in this suite. Other integrity rules, which restrict the structure of a thesaurus, are not covered in the constructs support suite, but in a specific integrity suite one. These integrity rules deal with the creation of structure by the addition of relationships between constructs, not with the support of constructs ${ }^{16}$. This paper only presents the suite of constructs.

This construct support suite is organized in two groups. First, there is a set of tests made for checking basic construct creation, presented in Table 1. Secondly, there are tests for the inclusion of relationships between constructs, which are listed in Table 2. The definition of each test under ISO 2788 and ISO 25964 is provided. When performing these tests, the equivalence with SKOS constructs (commented in Section 3) must be taken into account: SKOS tools will offer the creation of ConceptSchemes and Collections instead of Thesaurus or Concept groups, etc.

\footnotetext{
${ }^{16}$ Imagine a cycle of NT/BT relationships which express an inconsistency that is forbidden by one of these integrity rules. For example, a cycle expressing Female NT Person and Person NT Female would be inconsistent.
} 
Table 1. Tests for basic constructs.

\begin{tabular}{|l|l|l|}
\hline Test & ISO 2788 & ISO 25964 \\
\hline 1 & Create a Thesaurus & Create a Thesaurus \\
\hline 2 & Create a Domain & Create a concept group \\
\hline 3 & Create a Preferred Term & $\begin{array}{l}3.1 \text { Create a concept } \\
3.2 \text { Associate a Preferred Term to } \\
\text { the concept }\end{array}$ \\
\hline 4 & Create a Non-preferred Term & $\begin{array}{l}\text { Associate a Non-Preferred Term to } \\
\text { a concept }\end{array}$ \\
\hline 5 & Create a Note & Create a Note \\
\hline 6 & & Create an Array \\
\hline 7 & & Create a Term \\
\hline
\end{tabular}

Table 2. Tests for adding relationships between constructs.

\begin{tabular}{|c|c|c|}
\hline Test & ISO 2788 & ISO 25964 \\
\hline 8 & $\begin{array}{l}\text { Create a 'subdomain' (inclusion } \\
\text { between domains) relationship }\end{array}$ & $\begin{array}{l}\text { Create a } \\
\text { hasSuperGroup/hasSubGroup } \\
\text { relationship }\end{array}$ \\
\hline 9 & $\begin{array}{l}\text { Insert a Preferred Term in a } \\
\text { Domain }\end{array}$ & $\begin{array}{l}\text { Insert a Concept in a group of } \\
\text { concepts (property } \\
\text { ThesaurusConcept) }\end{array}$ \\
\hline 10 & $\begin{array}{l}\text { Create a BT relationship } \\
\text { between two Preferred Terms } \\
\text { that are not previously related }\end{array}$ & $\begin{array}{l}\text { Create a BT relationship between } \\
\text { two Concepts that are not } \\
\text { previously related: } \\
\text { a) without type } \\
\text { b) type = 'kind of' } \\
\text { c) type = 'part of' } \\
\text { d) type = 'instance of' }\end{array}$ \\
\hline 11 & $\begin{array}{l}\text { Create an NT relationship } \\
\text { between two Preferred Terms } \\
\text { that are not previously related }\end{array}$ & $\begin{array}{l}\text { Create an NT relationship between } \\
\text { two Concepts that are not } \\
\text { previously related: } \\
\text { a) without type } \\
\text { b) type = 'kind of' } \\
\text { c) type = 'part of' } \\
\text { d) type = 'instance of' }\end{array}$ \\
\hline 12 & $\begin{array}{l}\text { Create two BT/NT relationships } \\
\text { between a certain Preferred } \\
\text { Term and two different } \\
\text { ancestors (polyhierarchy) }\end{array}$ & $\begin{array}{l}\text { Create two BT/NT relationships } \\
\text { between a certain Concept and two } \\
\text { different ancestors }\end{array}$ \\
\hline 13 & $\begin{array}{l}\text { Create an RT relationship } \\
\text { between two Preferred Terms } \\
\text { not previously related }\end{array}$ & $\begin{array}{l}\text { Create an RT relationship between } \\
\text { two Concepts not previously } \\
\text { related }\end{array}$ \\
\hline 14 & Create a Top Term & Create a Top Concept \\
\hline 15 & & Insert a Concept in an Array \\
\hline
\end{tabular}

The possible values for the construct suite results are:

- M+I: Model implemented OK, with inference (reciprocal constructs are automatically included). 
- M: Model implemented OK, inference is not applicable. The construct is supported. No reciprocity requirements apply.

- M-I: Model implemented, but no inference is added (derived constructs are not automatically included). The construct is supported. Reciprocal constructs should be inserted by the tool, but they are not.

- PM: Problem for implementing the model (i.e., it is modeled, but incorrectly). The construct is supported, but it is not implemented in a correct manner.

- NM: No support for implementing the model (the construct is not modeled). The construct is not supported by the tool.

A summary of the construct test suite, with the possible results particularized for each test in the form of tables, is offered in the Appendix ${ }^{17}$. Changes were made to adapt the set of tests to ISO 25964. The most relevant changes concern tests that were not present in the previous version of the framework and are now introduced. In tables 1 and 2, there is a null value in the ISO 2788 column.

The introduction of two levels, Concepts and Terms, in ISO 25964 (in ISO 2788 there was only term) made it necessary to introduce a new test 7 , to create terms in a thesaurus. These terms can be associated to concepts with the hasPreferredLabel, etc. properties. It is worth remarking that this did not happen when the framework was first defined according to ISO 2788 in [23]. A preferred term in ISO 2788 and a SKOS concept were somehow equivalent: they were, respectively, the constructs that could participate in the basic relationships that a thesaurus can have. Now, only concepts participate. In addition, test 3 splits into two tests with ISO 25964. Test 3.1, for creating concepts, and test 3.2, for checking the possibility of adding several preferred terms to a concept, a characteristic of multilingual thesauri, which have a preferred term for each language.

Another remarkable novelty comes from the introduction of types in hierarchical relationships. Taking into account the emphasis that ISO 25964 puts on the distinction of the three types of BT/NT relationships, tests 10 and 11 have been expanded, so that the support of these relationships can be checked, but also the distinction between types. For similar reasons, the tests related with array support acquire more relevance with ISO 25964. In particular, the array creation (test 6) and the inclusion of concepts in arrays (test 15).

\subsection{Thesauri for Evaluation}

An ad-hoc thesaurus containing the minimal components needed to check construct support in thesaurus tools was created. There is no need for a bigger thesaurus, as one single test for each construct is enough to know if a tool provides the support checked. This thesaurus is a simplification, inspired by the EuroVoc thesaurus. It is necessary to emphasize that, as has been stated in the examples provided in Section 3, certain constructs incorporated into ISO 25964 have not been found in EuroVoc, and consequently, they have not been incorporated into this ad-hoc thesaurus either ${ }^{18}$.

\footnotetext{
${ }^{17}$ This detailed specification should be useful to get quick access to the test suite that can be applied. It does not substitute the content of section 4.2 , which shows its design.

${ }^{18}$ Regarding the proof of concept, it was not necessary to have a wider thesaurus; it was enough to verify if the tools had support for these constructs. Our priority has been to rely on a real thesaurus and no real thesauri have been found with all constructs.
} 
The ad-hoc thesaurus contains the constructs included in the tests: Domains (subject fields), Subdomains (microthesauri), PreferredTerms/Concepts, Non-PreferredTerms, Synonyms, Notes, BT/NT relationships, RT relationships, and polyhierarchy ${ }^{19}$. It includes two levels of hierarchical relationship (NT1 and NT2), shown in Figure 2(a). Geography is the subject field. The three subdomains (microthesauri) are preceded by the key MT in the figure. Top Terms are recognized by the TT keyword. A simpler version, in Figure 2(b), was prepared for use when polyhierarchy or several domain levels are not supported: this minimal version contains just two microthesauri, Europe and Regions of EU Member States, which eliminates the subject field (Geography) and the polyhierarchy (Spain only appears once as a narrower term, instead of twice). Both microthesauri are at the same level.

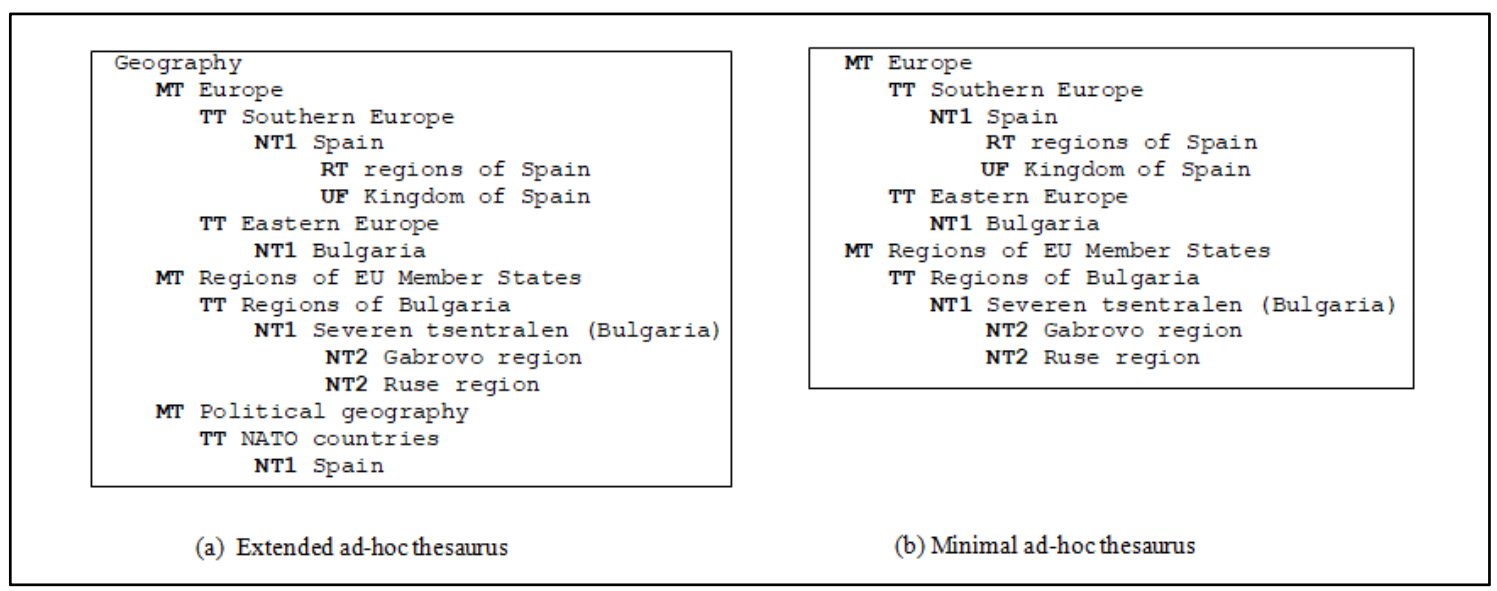

Figure 2. Ad-hoc thesaurus.

In addition, some other thesauri have been analyzed and imported to the tools used for the proof of concept. This was done to check that the constructs proposed in the ad-hoc thesaurus were those present in real thesauri. This acts as a guarantee that the ad-hoc thesaurus will be sufficiently representative of real thesauri for the types of constructs we consider (which include the most common types of constructs). They are the SKOS representation of the UKAT Thesaurus, the EuroVoc Thesaurus, and the NALT Thesaurus. They were successfully imported. However, it is worth remarking that all of them are represented with SKOS. Hence, the constructs included in ISO 25964 that have no equivalence in SKOS, e.g., compound equivalence, are not present. Moreover, these thesauri do not have compound equivalences. On the other hand, domain nesting, which occurs in both EuroVoc and UKAT, is represented in a different manner in both thesauri. Our analysis of this fact is carried out in Section 6.

\subsection{Indicators}

The results for each test offer the basis for measuring the quality of the support provided by the management thesaurus tool assessed, that is, the level of software conformance according to the standards. These measures can later be combined to obtain a set of indicators, which are measures that provide evaluations of specific attributes [32]. In this framework, the indicators proposed offer a measure of the quality of the support offered by the tools. Given that the aim of these indicators is to simplify the work of

\footnotetext{
${ }^{19}$ According to ISO 25964-1:2011, in a polyhierarchical structure, a single concept can occur in more than one place in the hierarchical structure of the thesaurus (p. 8).
} 
thesaurus editors in charge of selecting a thesaurus tool, it has been considered important to keep them simple and direct, easy to apply, and easy to understand. In the following, the base measures, together with the indicators derived from them, are presented.

A base measure is the translation to a numeric value, in the range [0-1], of the set of possible results for each test. The indicators defined from them will also take values in the same range. They are shown in Table 3 . When a tool models a construct perfectly, with rules of inference included, or when the inference is not applicable, the quality of the support is considered of maximal quality. Therefore, the result is assigned a value equal to 1 . The rest of possible results are mapped to numeric values according to the quality of the support offered: 0.5 for the M-I result, and 0.25 to the PM result. Finally, when there is no support, NM result, the equivalent is 0 .

Table 3. Values for quality support in tests.

\begin{tabular}{|l|c|c|}
\hline Semantic & Qualitative result & Numeric result \\
\hline Model implemented OK, with inference & M+I & 1 \\
\hline $\begin{array}{l}\text { Model implemented OK, inference is not } \\
\text { applicable }\end{array}$ & $\mathrm{M}$ & 1 \\
\hline Model implemented, but no inference is added & M-I & 0.5 \\
\hline Problem for implementing the model & PM & 0.25 \\
\hline No support for implementing the model & NM & 0 \\
\hline
\end{tabular}

The indicators proposed are aligned with the classification of tests into two sets: tests for basic constructs and for relationships. Therefore, they measure the quality of the support for basic constructs and the quality of the support for relationships, respectively. They can be obtained with similar formulas: the numeric values obtained in tests are added together and divided by the number of tests performed. Formulas (1) and (2) correspond, respectively, to the first one (quality of basic constructs) and the second one (quality of relationships). The number of tests performed is a decision of the person using the framework. For example, it is possible to choose to execute tests 10 and 11 as a single test, or to execute each variation, a), b), c), and d). This would depend on whether there is interest in testing the support of the three types of NT/BT relationships included in ISO 25964, or if testing the support of NT/BT, without distinctions, is enough.

Quality of basic constructs support $=\frac{\sum \text { (result of test for basic constructs) }}{\# \text { tests for basic constructs performed }}$

Quality of relationships support

$$
=\frac{\sum \text { (result of test for relationships) }}{\# \text { tests for relationships performed }}
$$

\section{Proof of concept}

The experimental validation has been performed on PoolParty and SKOSEd, two tools that use SKOS as the representation language for thesauri. Both tools have been chosen for this paper from the set of tools evaluated in [23] because the use of SKOS places them in an advantageous position to adapt to the new ISO 25964 standard, or at least be closer than other tools whose design was closer to ISO 2788. Despite there being more 
tools using SKOS for representing thesaurus, and new ones continue to join this list [38], no more tools have been included in this paper because the focus is on the framework definition and its revision to conform to ISO 25964; applying it to certain tools is a way to verify its feasibility.

PoolParty ${ }^{20}$ is a thesaurus tool with a Semantic Web approach [39]. It is a commercial software program with a free evaluation version that uses SKOS. The interface is designed to facilitate thesaurus edition. The version that was first evaluated in [23] was PoolParty 2.7. For this paper, the tests have been applied to PoolParty 5.

SKOSEd $^{21}$ is a Protégé plugin [40]. Its purpose is the creation and edition of KOS represented with SKOS. The reasoning is carried out through Protégé's reasoning facilities, for which the appropriate ontologies (the SKOS ontology in this case) have to be loaded onto Protégé. The possibilities offered by the loaded ontology will determine the support of the tool for some constructs and inferences. A main difference with PoolParty is, indeed, in the interface that users work with: it is Protégé, i.e., it is made for ontology engineers, and may be more overwhelming for thesaurus editors than that of PoolParty (e.g., the use of 'reasoners' to 'classify' items is quite specific to the ontology engineering area).

There is a relevant difference between the two tools selected: PoolParty is a thesaurus tool, while SKOSEd is, as its name indicates, a SKOS tool. This means that SKOSEd should support any type of KOS that complies with the SKOS recommendation, which is more flexible than thesaurus standards (see Section 3). However, restrictions specific to thesauri can be expected to be taken into account in PoolParty.

The construct suite has been checked using the thesaurus shown in Figure 2. The extended version, Figure 2(a), has been used. The thesaurus constructs supported by each tool are shown in Tables 4 and 5. The first column corresponds to the number of each test. In the second and third columns, a brief description of their semantics under ISO 2788 and ISO 25964 is offered (for a complete description, please refer to Section 4.2). The next columns show the results for each tool, with their qualitative and numeric values in each case.

Table 4. Support of basic constructs in PoolParty and SKOSEd.

\begin{tabular}{|l|l|l|c|c|c|c|}
\hline \multirow{2}{*}{ Test } & \multirow{2}{*}{ ISO 2788 } & \multirow{2}{*}{ ISO 25964 } & \multicolumn{2}{|c|}{ PoolParty } & \multicolumn{2}{c|}{ SKOSEd } \\
\cline { 3 - 6 } & & Results & Values & Results & Values \\
\hline 1 & Thesaurus & Thesaurus & $\mathrm{M}$ & 1 & $\mathrm{M}$ & 1 \\
\hline 2 & Domain & Concept group & $\mathrm{M}-\mathrm{I}$ & 0.5 & $\mathrm{NM}$ & 0 \\
\hline 3 & Preferred Term & $\begin{array}{l}3.1 \text { Concept } \\
\text { 3.2 Associate Pre- } \\
\text { ferredTerm }\end{array}$ & $\mathrm{M}$ & 1 & $\mathrm{M}$ & 1 \\
\hline 4 & $\begin{array}{l}\text { Non-preferred } \\
\text { Term }\end{array}$ & $\begin{array}{l}\text { Associate NonPre- } \\
\text { ferredTerm to a concept }\end{array}$ & $\mathrm{M}$ & 1 & $\mathrm{M}$ & 1 \\
\hline 5 & Note & Note & $\mathrm{M}$ & 1 & $\mathrm{M}$ & 1 \\
\hline 6 & & Array & $\mathrm{M}$ & 1 & $\mathrm{NM}$ & 0 \\
\hline 7 & & Term & & & & \\
\hline
\end{tabular}

${ }^{20} \mathrm{https}: / /$ www.poolparty.biz/

${ }^{21} \mathrm{https} / /$ code.google.com/archive/p/skoseditor/ 
Table 5. Support of relationships in PoolParty and SKOSEd.

\begin{tabular}{|c|c|c|c|c|c|c|}
\hline \multirow{2}{*}{ Test } & \multirow{2}{*}{ ISO 2788} & \multirow{2}{*}{ ISO 25964} & \multicolumn{2}{|c|}{ PoolParty } & \multicolumn{2}{|c|}{ SKOSEd } \\
\hline & & & Results & Values & Results & Values \\
\hline 8 & Subdomain & $\begin{array}{l}\text { hasSuperGroup/has- } \\
\text { SubGroup }\end{array}$ & NM & 0 & NM & 0 \\
\hline 9 & $\begin{array}{l}\text { Preferred Term } \\
\text { in Domain }\end{array}$ & $\begin{array}{l}\text { Concept in Concept } \\
\text { group }\end{array}$ & M-I & 0.5 & M-I & 0.5 \\
\hline 10 & BT relationship & $\begin{array}{l}\text { BT relationships: } \\
\text { a) without type } \\
\text { b) type = 'kind of' } \\
\text { c) type = 'part of' } \\
\text { d) type = 'instance of' }\end{array}$ & $\begin{array}{l}\mathrm{M}+\mathrm{I} \\
\mathrm{NM} \\
\mathrm{NM} \\
\mathrm{NM}\end{array}$ & $\begin{array}{l}1 \\
0 \\
0 \\
0\end{array}$ & $\begin{array}{l}\mathrm{M}+1 \\
\mathrm{NM} \\
\mathrm{NM} \\
\mathrm{NM}\end{array}$ & $\begin{array}{l}1 \\
0 \\
0 \\
0\end{array}$ \\
\hline 11 & NT relationship & $\begin{array}{l}\text { NT relationship: } \\
\text { a) without type } \\
\text { b) type = 'kind of' } \\
\text { c) type = 'part of' } \\
\text { d) type = 'instance of' }\end{array}$ & $\begin{array}{l}\mathrm{M}+\mathrm{I} \\
\mathrm{NM} \\
\mathrm{NM} \\
\mathrm{NM}\end{array}$ & $\begin{array}{l}1 \\
0 \\
0 \\
0\end{array}$ & $\begin{array}{l}\mathrm{M}+\mathrm{I} \\
\mathrm{NM} \\
\mathrm{NM} \\
\mathrm{NM}\end{array}$ & $\begin{array}{l}1 \\
0 \\
0 \\
0\end{array}$ \\
\hline 12 & Polyhierarchy & Polyhierarchy & $\mathrm{M}+\mathrm{I}$ & 1 & $\mathrm{M}+\mathrm{I}$ & 1 \\
\hline 13 & RT relationship & RT relationship & $\mathrm{M}+\mathrm{I}$ & 1 & $\mathrm{M}+\mathrm{I}$ & 1 \\
\hline 14 & Top Term & Top Concept & $\mathrm{M}+\mathrm{I}$ & 1 & $\mathrm{M}+\mathrm{I}$ & 1 \\
\hline 15 & & Concept in Array & $\mathrm{M}+\mathrm{I}$ & 1 & $\mathrm{NM}$ & 0 \\
\hline
\end{tabular}

PoolParty works with SKOS constructs: ConceptSchemes, Concepts, and Labels. It infers reciprocal relationships when creating BT, NT or RT relationships. SKOS Collections are supported. Thus, they can be used to represent concept groups (domains) and arrays, as proposed in [18].

PoolParty has perfect support for polyhierarchy (e.g., it has been possible to create, without problems, the polyhierarchical structure of the thesaurus in Figure 2(a)), but it does not support domain nesting tested in test 8 , i.e., it only permits one level of ConceptSchemes. Regarding annotation, PoolParty only supports Scope notes. The new updates of ISO 25964, such as types in hierarchical relationships, are not supported. The values obtained for the indicators are presented in the second column of Table 6.

SKOSEd also works with SKOS constructs. An important difference with PoolParty in the constructs supported is that there is no SKOSEd view to add collections. This implies that it is not possible to represent domains or arrays with collections as proposed in [18]. In consequence, the results for tests 2, 6 and 15 differ from those of PoolParty.

In SKOSEd the inference of reciprocal relationships is not automatically done when a semantic relationship is added, but it is offered when the 'Classify' reasoning facility is performed. Polyhierarchy is supported. Only one level of ConceptSchemes is accepted by the tool, i.e., domain nesting is not supported. The values obtained for the indicators are shown in the third column of Table 6. 
Both tools evidence a reasonable support for the constructs used in most thesauri ${ }^{22}$. PoolParty offers better support, as the values in Table 6 show. This is logical, as this tool, by contrast to SKOSEd, was conceived as a thesaurus tool.

Moreover, some constructs that appeared with ISO 25964 are not supported (see the results of tests 6,10 and 11). Some more considerations derived from the results of this experimental validation are offered in the Analysis Section (Section 6).

Table 6. Values obtained for the indicators in the experimental validation.

\begin{tabular}{|l|c|c|}
\hline Indicator & PoolParty & SKOSEd \\
\hline Quality of basic constructs support & $\frac{6.5}{8}=0.8$ & $\frac{5}{8}=0.6$ \\
\hline Quality of relationships support & $\frac{6.5}{14}=0.5$ & $\frac{5.5}{14}=0.4$ \\
\hline
\end{tabular}

\section{Analysis}

The most interesting remarks are related to constructs that are new in ISO 25964, which were not in ISO 2788, and have no equivalence in SKOS. The following comments affect tests that check the support of 1) BT/NT relationships, 2) Subdomains, 3) Collections and 4) Polyhierarchy.

The expansion of tests 10 and 11 to cover the possibility of assigning types to BT/NT relationships has been made with subtests, numbered 10.a), 10.b), etc. It has been decided to do it in this way to provide the framework with flexibility. Thus, if a user considers the support of types in BT/NT relationships to be important, the four variations will be applied as independent tests, that is, four tests will be executed and the indicators will be adapted appropriately. However, users who consider the support of types in BT/NT relationships to be irrelevant can maintain the a) variation, "no type", which corresponds to the possibility already available in ISO 2788 , and the one used in most thesauri we have observed. In the experimental validation of Section 5, we worked with the first approach, so the number of tests considered to calculate the indicator for the relationships support is 14 (see the last row of Table 6).

Regarding the implications that the introduction of types of BT/NT relationships has, it is worth taking into account the fact that the manner of representing the diversity of hierarchies associated to types of BT/NT relationships in SKOS has no definitive solution yet (see the proposals made in $[18,36]$ ). In our opinion, this implies that the tools will not support these types until everything concerning their representation with SKOS and their management is stabilized. Given that the extensions to SKOS that would support the distinction between the different types of BT/NT relationships is not yet included in the SKOS recommendation, our prevision is that most tools will fail in tests that check the support of these new constructs. They are created with the intention of being available when stabilization arrives.

We remark that the support of subdomains was already included in the previous version of this framework, as well as the support of nesting between them. There is no equivalent property in SKOS for ISO 25964 hasSuperGroup/hasSubGroup. This

\footnotetext{
22 Besides using the ad-hoc thesaurus presented in section 4.3, some real thesauri were imported (the reasons that motivated the examination of these thesauri are presented in section 4.3). The analysis made by comparing the manner each of them represents thesaurus constructs with SKOS is presented in section 6 .
} 
introduces a level of uncertainty that will not encourage software developers to include support for this capability (which was not necessary with ISO 2788) in their tools. This situation is similar to that described about types in hierarchical relationships.

The support for subdomains seems to be a pending task. So, when a thesaurus has this structure (e.g., the EuroVoc Thesaurus) one solution is that found in the UKAT Thesaurus: not to represent the top level of subject fields and start with the representation of subdomains (microthesauri) as ConceptSchemes, which are placed at the top level of the hierarchy. Another possibility, which is the one that probably comes to mind when thesaurus tools are used to create a thesaurus (these tools do not support two levels of ConceptSchemes), is to use Concepts at all levels, even to represent domains. These are not ideal solutions, but until the mapping of ISO 25964 and SKOS gets fixed, we are afraid this situation will not change. Finally, there are other possibilities based on extending SKOS with OWL properties defined ad-hoc to represent this semantic. This solution was chosen for the EuroVoc representation available at the EuroVoc official web page ${ }^{23}$. The drawback of using ad-hoc extensions is that they are not understood by generic thesaurus tools.

Testing two different tools, one of them oriented to thesauri and the other focused on SKOS, has helped to corroborate the interest of comparing the constructs of ISO 25964 and SKOS. In fact, some results of the experiments on these tools (differences in results of some tests) had been foreseen as possible, due to the comparison discussed in Section 3. Moreover, thanks to that comparative analysis, an explanation of the reasons for these differences was already available.

A difference was found in the support of collections by the tools assessed in Section 5 (compare the results of tests 2, 6, and 15 in Tables 4 and 5). With ISO 2788, the role collections could have for representing a thesaurus was minor. However, with ISO 25964, the fact that collections are proposed as the SKOS equivalent for concept groups and arrays, means that they acquire relevance. This difference between tools, which was almost irrelevant before ISO 25964, increases its relevance with the new standard.

It is positive to verify that polyhierarchy is well modeled and supported in the tools assessed in the experimental validation. Even if not common, polyhierarchy is a structure used in some thesauri (e.g., EuroVoc has some examples). It is good news for thesaurus editors to have this support when needed.

The experiments have confirmed the suspected limitations with new constructs and structures in ISO25964, or those which acquire a greater relevance than they had in ISO 2788: collections, terms, polyhierarchy, arrays, compound equivalence, etc. This may be due to what is evidenced in [37]: the special complexity that ISO 25964 introduces with respect to SKOS, e.g., with the possibility it introduces to relate concepts and terms with isNonPreferredLabelFor/isPreferredLabelFor relationships.

\section{Conclusions}

This paper presents a methodological framework to assess construct support in thesaurus tools, whose target community is thesauri editors concerned with obtaining thesauri that conform to pertinent thesauri standards. It is oriented towards the design of thesaurus management tools and their users' experience. Construct support is a crucial

\footnotetext{
${ }^{23}$ https://publications.europa.eu/en/web/eu-vocabularies/th-dataset/-/resource/dataset/eurovoc
} 
issue when creating and editing thesauri. Thus, the help provided by tools in detecting possible infractions of the standard and preventing them is valuable, as this will contribute to the quality of the thesauri created with them. Being able to verify the level of support that can be obtained from a prospective tool to be used for thesaurus editing can itself be helpful for thesaurus editors confronted with the task of selecting a tool. This is what is offered in this framework. In addition, this work can be relevant for SKOS or thesaurus developers interested in testing their abilities, applying a SKOS tool to maintain, or publish, a thesaurus in the Semantic Web or vice versa.

The proposal presented in this paper is part of a generic framework, which includes functionality and other issues, besides the one presented here [23]. Moreover, in this paper, the comparison between the ISO standards for thesauri and the SKOS recommendation is deployed. The changes that ISO 25964 introduces with respect to ISO 2788 and the modifications it implies on this methodological framework have been analyzed. The inclusion of SKOS and ISO 25964 is a relevant novelty of this proposal as compared with some previous studies related to the evaluation of thesauri or thesauri tools [27-29]. This proposal was first designed taking into account the ISO 2788 standard, still supported by thesaurus tools, and revised conforming to ISO 25964 . It was important to keep both perspectives in parallel, as a complete adaptation of thesauri and tools to ISO 25964 may be a long process. The future will show how thesauri tools adapt to the new ISO standard.

This proposal is specifically made for thesauri tools, which differentiates it from general Semantic Web benchmarks [30], even if they have been taken into account for its definition and specification. In this framework, thesauri editors can find a guide, not otherwise available, for comparing thesaurus tools, that they can apply directly. The proposed model is systematic and the indicators offer a means to measure and compare the conformance of thesaurus management software with respect to the necessary support in managing the constructs.

Tests for compound equivalences and some other constructs which are new in ISO 25964 have not been included in Tables 1 and 2. The reason is that we have not found thesauri with such constructs, which implies that, if thesaurus designers do not use these constructs, neither will they be interested in checking if thesaurus tools are able to manage them. However, their inclusion would not affect the suite, as it would just add new tests without modifying the available ones.

Even if SKOS is used by the tools evaluated to represent concept-based thesauri, this proposal is not conceived for SKOS tools, but for thesauri tools. A proposal for SKOS tools should have different tests and a different set of results, with different issues to take into consideration (remember, e.g., the analysis related to types of hierarchical relationships, and subdomains). However, the analysis of the correspondences between ISO 25964 constructs and SKOS constructs is a valuable tool to understand how the tests can be applied to any thesaurus tool. The analysis carried out shows that some characteristics of thesauri are not well supported when SKOS is used for their representation.

The comparison of the solutions adopted in various thesauri to represent the constructs that do not find direct equivalence in SKOS, compound concepts, domain nesting, etc., shows that, in these cases, the editors of thesauri are the ones who assume the responsibility to decide the mapping of ISO constructs to SKOS constructs. Evidence focused on the solution found for domain nesting in the UKAT Thesaurus and in EuroVoc (see Section 6) has been offered. Naturally, the disadvantage of these solutions 
is that they are not standard. Proposals to extend SKOS to support these thesauri constructs, such as ISO-THES, have been carried out. However, the thesaurus management tools do not integrate support for ISO-THES, which implies that the responsibility for transferring these proposals adequately to their thesauri continues to fall to the editors of the thesauri. In our opinion, it would be necessary for proposals such as ISO-THES to evolve to the W3C Recommendation status. This would allow tool developers to provide support by default, thus facilitating the task of the professionals in charge of thesauri edition.

\section{References}

[1] F.-J. Garcia-Marco, The evolution of thesauri and the history of knowledge organization: between the sword of mapping knowledge and the wall of keeping it simple, Brazilian J. Inf. Sci. Res. Trends. 10 (2016) 10-11. doi:10.5016/brajis.v10i1.5786.

[2] F.-J. García-Marco, Enhancing the Visibility and Relevance of Thesauri in the Web: Searching for a Hub in the Linked Data Environment., Knowl. Organ. 43 (2016) 193-202.

[3] A. Isaac, T. Baker, Linked data practice at different levels of semantic precision: The perspective of libraries, archives and museums, Bull. Am. Soc. Inf. Sci. Technol. 41 (2015) 34-39. doi:10.1002/bult.2015.1720410411.

[4] A. Mastora, M. Peponakis, S. Kapidakis, SKOS concepts and natural language concepts: An analysis of latent relationships in KOSs, J. Inf. Sci. 43 (2017) 492508. doi:10.1177/0165551516648108.

[5] M. Hildebrand, J. van Ossenbruggen, L. Hardman, G. Jacobs, Supporting subject matter annotation using heterogeneous thesauri: A user study in Web data reuse, Int. J. Hum. Comput. Stud. 67 (2009) 887-902. doi:10.1016/J.IJHCS.2009.07.008.

[6] ISO, ISO 25964-1:2011 Information and Documentation -Thesauri and Interoperability with Other Vocabularies -Part 1: Thesauri for Information Retrieval, International Organization for Standardization, 2011.

[7] N.F. Noy, M. Sintek, S. Decker, M. Crubezy, R.W. Fergerson, M.A. Musen, Creating Semantic Web contents with Protege-2000, IEEE Intell. Syst. 16 (2001) 60-71. doi:10.1109/5254.920601.

[8] ISO, ISO 2788:1986 Documentation Guidelines for the Establishment and Development of Monolingual Thesauri, 2nd ed., International Organization for Standardization, 1986.

[9] ISO, ISO 5964:1985. Guidelines for the establishment and development of multilingual thesauri, International Organization for Standardization, 1985.

[10] ISO, ISO 25964-2:2011 Information and Documentation -Thesauri and Interoperability with Other Vocabularies -Part 2: Interoperability with other vocabularies, International Organization for Standardization, 2013.

[11] S.G.D. Clarke, M.L. Zeng, From ISO 2788 to ISO 25964 : The evolution of thesaurus standards towards interoperability and data modeling, Inf. Stand. Q. 24 (2012) 20-26. doi:http://dx.doi.org/10.3789/isqv24n1.2012.04. 
[12] J.-A. Pastor-Sánchez, Proposal to represent the UNESCO thesaurus for the Semantic Web applying ISO 25964, Brazilian J. Inf. Sci. Res. Trends. 9 (2015) 1-8. http://www2.marilia.unesp.br/revistas/index.php/bjis/article/view/5684/3897 (accessed May 18, 2018).

[13] I. Jurisica, J. Mylopoulos, E. Yu, Ontologies for Knowledge Management: An Information Systems Perspective, Knowl. Inf. Syst. 6 (2004) 380-401. doi:10.1007/s10115-003-0135-4.

[14] A. Miles, S. Bechhofer, SKOS Simple Knowledge Organization System Reference, World Wide Web Consortium, 2009. https://www.w3.org/TR/skosreference/ (accessed May 18, 2018).

[15] T. Baker, S. Bechhofer, A. Isaac, A. Miles, G. Schreiber, E. Summers, Key choices in the design of Simple Knowledge Organization System (SKOS), Web Semant. Sci. Serv. Agents World Wide Web. 20 (2013) 35-49. doi:10.1016/J.WEBSEM.2013.05.001.

[16] A.J. O'dell, The Visual Vocabulary: Skos:example and the Illustrated $<\mathrm{i}>$ Artists' Books Thesaurus $</ i>$, J. Libr. Metadata. 15 (2015) 241-251. doi:10.1080/19386389.2015.1103086.

[17] A. Isaac, E. Summers, SKOS Simple Knowledge Organization System Primer, 2009. https://www.w3.org/TR/skos-primer/ (accessed May 18, 2018).

[18] A. Isaac, Correspondence between ISO 25964 and SKOS/SKOS-XL models, 2013.

https://groups.niso.org/apps/group_public/download.php/12351/Correspondence ISO25964-SKOSXL-MADS-2013-12-11.pdf.

[19] B.C. Villazón-Terrazas, M.C. Suárez-Figueroa, A. Gómez-Pérez, A PatternBased Method for Re-Engineering Non-Ontological Resources into Ontologies, Int. J. Semant. Web Inf. Syst. 6 (2010) 27-63.

[20] D. Kless, S. Milton, E. Kazmierczak, Relationships and relata in ontologies and thesauri: Differences and similarities, Appl. Ontol. 7 (2012) 401-428. doi:10.3233/AO-2012-0118.

[21] D. Kless, S. Milton, E. Kazmierczak, J. Lindenthal, Thesaurus and ontology structure: Formal and pragmatic differences and similarities, J. Assoc. Inf. Sci. Technol. 66 (2015) 1348-1366. doi:10.1002/asi.23268.

[22] E. Francesconi, Semantic model for legal resources: Annotation and reasoning over normative provisions, Semant. Web. 7 (2016) 255-265. doi:10.3233/SW140150 .

[23] M.M. Martínez-González, M.-L. Alvite-Díez, On the evaluation of thesaurus tools compatible with the Semantic Web, J. Inf. Sci. 40 (2014) 711-722. doi: $10.1177 / 0165551514545603$.

[24] B. Kitchenham, S.L. Pfleeger, N. Fenton, Towards a framework for software measurement validation, IEEE Trans. Softw. Eng. 21 (1995) 929-944. doi:10.1109/32.489070.

[25] A.R. Weiss, Dhrystone Benchmark: History, Analysis, Scores and Recommendations. White paper, EEMBC Certifcation Laboratories, LLC, Austin, 2002. 
[26] O. Suominen, C. Mader, Assessing and Improving the Quality of SKOS Vocabularies, 3 (2014) 47-73. doi:10.1007/s13740-013-0026-0.

[27] F. Severino, The Term Development in the Thesauri of International Organisations, Eur. J. Dev. Res. 19 (2007) 327-351. doi:10.1080/09578810701289261.

[28] G. Moya Martínez, I. Gil Leiva, Evaluación de softwares de gestión de tesauros, Ciencias La Inf. 32 (2001) 3-23.

[29] Willpower Information, Software for building and editing thesauri, (2014). http://www.willpowerinfo.co.uk/thessoft.htm (accessed May 18, 2018).

[30] R. García-Castro, A. Gómez-Pérez, Benchmarking in the Semantic Web, in: Semant. Web Eng. Knowl. Soc., IGI Global, 2009: pp. 341-370. doi:10.4018/978-1-60566-060-8.ch200.

[31] M. Poveda-Villalón, A. Gómez-Pérez, M.C. Suárez-Figueroa, OOPS! (OntOlogy Pitfall Scanner!): supporting ontology evaluation on-line, Int. J. Semant. Web Inf. Syst. 10 (2014) 7-34. doi:10.4018/ijswis.2014040102.

[32] F. Radulovic, R. García-Castro, A. Gómez-Pérez, SemQuaRE - An extension of the SQuaRE quality model for the evaluation of semantic technologies, Comput. Stand. Interfaces. 38 (2015) 101-112. doi:10.1016/J.CSI.2014.09.001.

[33] A. Miles, S. Bechhofer, SKOS Simple Knowledge Organization System eXtension for Labels (SKOS-XL) Namespace Document - HTML Variant, World Wide Web Consortium, 2009. https://www.w3.org/TR/skosreference/skos-xl.html.

[34] A. Isaac, E. De Smedt, Note apply to the published mapping between the ISO 25964 data model and the SKOS schema, (2015).

http://pub.tenforce.com/schemas/iso25964/skosthes.

[35] L. Will, The ISO 25964 data model for the structure of an information retrieval thesaurus, Bull. Am. Soc. Inf. Sci. Technol. 38 (2012) 48-51. doi:10.1002/bult.2012.1720380413.

[36] V. Alexiev, A. Isaac, J. Lindenthal, On the composition of ISO 25964 hierarchical relations (BTG, BTP, BTI), Int. J. Digit. Libr. 17 (2016) 39-48. doi:10.1007/s00799-015-0162-2.

[37] J.-A. Pastor-Sánchez, ISO-THES: Ampliando Skos a partir de la norma de tesauros ISO 25964, Anu. ThinkEPI. 7 (2013) 189-193.

[38] A. Stellato, S. Rajbhandari, A. Turbati, M. Fiorelli, C. Caracciolo, T. Lorenzetti, J. Keizer, M.T. Pazienza, VocBench: A Web Application for Collaborative Development of Multilingual Thesauri, in: Z.A. Gandon F., Sabou M., Sack H., d'Amato C., Cudré-Mauroux P. (Ed.), Semant. Web. Latest Adv. New Domains. ESWC 2015. Lect. Notes Comput. Sci. Vol 9088, Springer, 2015: pp. 38-53. doi:10.1007/978-3-319-18818-8_3.

[39] T. Schandl, A. Blumauer, PoolParty: SKOS Thesaurus Management Utilizing Linked Data, in: Aroyo L. et al. (Ed.), Semant. Web Res. Appl. ESWC 2010. Lect. Notes Comput. Sci. Vol 6089, Springer, Berlin, Heidelberg, 2010: pp. 421425. doi:10.1007/978-3-642-13489-0_36.

[40] S. Jupp, S. Bechhofer, R. Stevens, A Flexible API and Editor for SKOS, in: 
Aroyo L. et al. (Ed.), Semant. Web Res. Appl. ESWC 2009. Lect. Notes Comput. Sci. Vol 5554, Springer-Verlag, Berlin, Heidelberg, 2009: pp. 506-520. doi:doi.org/10.1007/978-3-642-02121-3_38. 


\section{APPENDIX - CONSTRUCT SUPPORT SUITE}

Table 1. Set of possible values for test results.

\begin{tabular}{|l|l|l|}
\hline (RESULT) CODE & (RESULT) VALUE & DESCRIPTION \\
\hline M+I & $\begin{array}{l}\text { Model implemented OK, with } \\
\text { inference }\end{array}$ & $\begin{array}{l}\text { Construct supported. } \\
\text { Reciprocal constructs are } \\
\text { inserted. }\end{array}$ \\
\hline M & $\begin{array}{l}\text { Model implemented OK, } \\
\text { inference is not applicable }\end{array}$ & $\begin{array}{l}\text { Construct supported. No } \\
\text { reciprocity requirements } \\
\text { apply. }\end{array}$ \\
\hline M-I & $\begin{array}{l}\text { Model implemented, but no } \\
\text { inference is added }\end{array}$ & $\begin{array}{l}\text { Construct supported. } \\
\text { Reciprocal constructs should } \\
\text { be inserted by the tool, but } \\
\text { they are not. }\end{array}$ \\
\hline PM & $\begin{array}{l}\text { Problem for implementing the } \\
\text { model }\end{array}$ & $\begin{array}{l}\text { Construct supported, but it is } \\
\text { not implemented in a correct } \\
\text { manner }\end{array}$ \\
\hline NM & $\begin{array}{l}\text { No support for implementing } \\
\text { the model }\end{array}$ & $\begin{array}{l}\text { Construct not supported by the } \\
\text { tool }\end{array}$ \\
\hline
\end{tabular}

Table 2. Tests for basic constructs.

\begin{tabular}{|l|l|l|l|}
\hline Test & ISO 2788 & ISO 25964 & Possible results \\
\hline 1 & Create a Thesaurus & Create a Thesaurus & $\begin{array}{l}\text { M: Thesaurus supported } \\
\text { NM: No support for thesaurus construct }\end{array}$ \\
\hline 2 & Create a Domain & $\begin{array}{l}\text { Create a concept } \\
\text { group }\end{array}$ & $\begin{array}{l}\text { M+I: Domain/Concept group supported. } \\
\text { The tool checks that it has been placed at } \\
\text { the correct level in the thesaurus } \\
\text { structure (on top of concepts). } \\
\text { M-I: Domain/Concept group supported. } \\
\text { No checks of its position in thesaurus } \\
\text { structure. } \\
\text { NM: No support for domains/concept } \\
\text { groups. }\end{array}$ \\
& Term & $\begin{array}{l}\text { Create a Preferred } \\
\text { Concept }\end{array}$ & $\begin{array}{l}\text { M: Preferred Term/Concept supported. } \\
\text { NM: Preferred Term/Concepts are not } \\
\text { supported. }\end{array}$ \\
\hline 3 & & $\begin{array}{l}\text { 3.2 Associate a } \\
\text { PreferredTerm to }\end{array}$ & M: An additional PreferredTerm can be \\
\hline
\end{tabular}




\begin{tabular}{|l|l|l|l|}
\hline & & the concept & $\begin{array}{l}\text { linked to a concept. } \\
\text { NM: The tool does not support the } \\
\text { addition of more than one Preferred } \\
\text { Term for each concept, even if the } \\
\text { language differs. }\end{array}$ \\
\hline 4 & $\begin{array}{l}\text { Create a Non- } \\
\text { Preferred Term }\end{array}$ & $\begin{array}{l}\text { Associate a Non- } \\
\text { Preferred Term to a } \\
\text { concept }\end{array}$ & $\begin{array}{l}\text { M: An additional NonPreferredTerm can } \\
\text { be linked to a concept. } \\
\text { NM: The tool does not support the } \\
\text { addition of NonPreferredTerm to a } \\
\text { concept. }\end{array}$ \\
\hline 5 & Create a Note & Create a Note & $\begin{array}{l}\text { M: Notes can be added to thesaurus } \\
\text { constructs. } \\
\text { NM: Notes are not supported. }\end{array}$ \\
\hline 6 & Create an Array & $\begin{array}{l}\text { M: Arrays can be created by grouping } \\
\text { concepts. } \\
\text { NM: Arrays are not supported. }\end{array}$ \\
\hline 7 & Create a Term & $\begin{array}{l}\text { M: Terms are supported. A type of } \\
\text { construct is available for Terms. } \\
\text { NM: There is no construct type for } \\
\text { Terms. }\end{array}$ \\
\hline
\end{tabular}

Table 3. Tests for adding relationships between constructs.

\begin{tabular}{|l|l|l|l|}
\hline Test & ISO 2788 & ISO 25964 & Possible results \\
\hline 8 & $\begin{array}{l}\text { Create a } \\
\text { 'subdomain' } \\
\text { (inclusion between } \\
\text { domains) } \\
\text { relationship }\end{array}$ & $\begin{array}{l}\text { Create a } \\
\text { hasSuperGroup/has } \\
\text { SubGroup } \\
\text { relationship }\end{array}$ & $\begin{array}{l}\text { M+I: It is possible to have several levels } \\
\text { of Domains (to relate two Domains with } \\
\text { a relationship). The tool inserts the } \\
\text { reciprocal relationship. } \\
\text { M-I: It is possible to relate two Domains } \\
\text { with a relationship. The tool does not } \\
\text { insert the reciprocal relationship. } \\
\text { PM: } \\
\text { NM: It is not possible to have several }\end{array}$ \\
& $\begin{array}{l}\text { Insert a Preferred } \\
\text { Term in a Domain } \\
\text { levels of Domains. }\end{array}$ \\
& $\begin{array}{l}\text { Insert a Concept in } \\
\text { agroup of concepts }\end{array}$ & $\begin{array}{l}\text { M+I: It is possible to insert a } \\
\text { PreferredTerm/Concept in a } \\
\text { Domain/Group of Concepts } \\
\text { (isMemberOfGroup/hasAsMember } \\
\text { property). The tool does not insert the } \\
\text { reciprocal relationship. }\end{array}$ \\
\hline
\end{tabular}




\begin{tabular}{|c|c|c|c|}
\hline & & & $\begin{array}{l}\text { M-I: It is possible to insert a } \\
\text { PreferredTerm/Concept in a } \\
\text { Domain/Group of Concepts. The tool } \\
\text { does not insert the reciprocal } \\
\text { relationship. } \\
\text { NM: It is not possible to insert } \\
\text { PreferredTerm/Concept in a } \\
\text { Domain/Group of Concepts. }\end{array}$ \\
\hline 10 & $\begin{array}{l}\text { Create a BT } \\
\text { relationship } \\
\text { between two } \\
\text { Preferred Terms } \\
\text { that are not } \\
\text { previously related }\end{array}$ & $\begin{array}{l}\text { Create a BT } \\
\text { relationship } \\
\text { between two } \\
\text { Concepts that are } \\
\text { not previously } \\
\text { related: } \\
\text { e) without type } \\
\text { f) type = 'kind } \\
\text { of' } \\
\text { g) type = 'part of' } \\
\text { h) type = } \\
\text { 'instance of' }\end{array}$ & $\begin{array}{l}\text { M+I: It is possible to create a BT } \\
\text { relationship --of the chosen type, a), b), } \\
\text { c), d)-- between two concepts. The tool } \\
\text { inserts the reciprocal relationship (NT). } \\
\text { M-I: It is possible to create a BT } \\
\text { relationship between two concepts. The } \\
\text { tool does not insert the reciprocal } \\
\text { relationship. } \\
\text { NM: It is not possible to create a BT } \\
\text { relationship between two concepts. }\end{array}$ \\
\hline 11 & $\begin{array}{l}\text { Create an NT } \\
\text { relationship } \\
\text { between two } \\
\text { Preferred Terms } \\
\text { that are not } \\
\text { previously related }\end{array}$ & $\begin{array}{l}\text { Create an NT } \\
\text { relationship } \\
\text { between two } \\
\text { Concepts that are } \\
\text { not previously } \\
\text { related: } \\
\text { a) without type } \\
\text { b) type = 'kind } \\
\text { of' } \\
\text { c) type = 'part of' } \\
\text { d) type = } \\
\text { 'instance of' }\end{array}$ & $\begin{array}{l}\text { M+I: It is possible to create an NT } \\
\text { relationship --of the chosen type, a), b), } \\
\text { c), d)-- between two concepts. The tool } \\
\text { inserts the reciprocal relationship (BT). } \\
\text { M-I: It is possible to create a BT } \\
\text { relationship between two concepts. The } \\
\text { tool does not insert the reciprocal } \\
\text { relationship. } \\
\text { NM: It is not possible to create an NT } \\
\text { relationship between two concepts. }\end{array}$ \\
\hline 12 & $\begin{array}{l}\text { Create two BT/NT } \\
\text { relationships } \\
\text { between a certain } \\
\text { Preferred Term and } \\
\text { two different } \\
\text { ancestors } \\
\text { (polyhierarchy) }\end{array}$ & $\begin{array}{l}\text { Create two BT/NT } \\
\text { relationships } \\
\text { between a certain } \\
\text { Concept and two } \\
\text { different ancestors }\end{array}$ & $\begin{array}{l}\text { M+I: It is possible to create an NT } \\
\text { relationship between a concept A and a } \\
\text { second one, B (A NT B), even if A was } \\
\text { already linked to another concept C by } \\
\text { an NT relationship (A NT C). The tool } \\
\text { inserts the reciprocal relationship (B BT } \\
\text { A) or it is possible to create a BT } \\
\text { relationship between a concept B and a } \\
\text { concept A, (B BT A), even if A was } \\
\text { already linked to another concept C by a } \\
\text { BT relationships (B BT A). } \\
\text { M-I: It is possible to create an NT } \\
\text { relationship between a concept A and a } \\
\text { second one, B (A NT B), even if A was } \\
\text { already linked to another concept C by } \\
\text { an NT relationship (A NT C). The tool } \\
\text { does not insert the reciprocal relationship }\end{array}$ \\
\hline
\end{tabular}




\begin{tabular}{|c|c|c|c|}
\hline & & & $\begin{array}{l}\text { (BT). } \\
\text { PM: It is possible to create an NT } \\
\text { relationship between a concept A and a } \\
\text { second one, B (A NT B), even if A was } \\
\text { already linked to another concept C by } \\
\text { an NT relationship (A NT C). However, } \\
\text { the structure implemented is not the one } \\
\text { intended (e.g., some concepts are not } \\
\text { placed at the correct level they should be } \\
\text { on with respect to the other two) } \\
\text { NM: It is not possible to create an NT } \\
\text { relationship between a concept A and a } \\
\text { second one, B (A NT B), even if A was } \\
\text { already linked to another concept C by } \\
\text { an NT relationship (A NT C). }\end{array}$ \\
\hline 13 & $\begin{array}{l}\text { Create an RT } \\
\text { relationship } \\
\text { between two } \\
\text { Preferred Terms } \\
\text { not previously } \\
\text { related }\end{array}$ & $\begin{array}{l}\text { Create an RT } \\
\text { relationship } \\
\text { between two } \\
\text { Concepts not } \\
\text { previously related }\end{array}$ & $\begin{array}{l}\text { M+I: It is possible to create an RT } \\
\text { relationship between two concepts (A RT } \\
\text { B). The tool inserts the reciprocal } \\
\text { relationship (B RT A). } \\
\text { M-I: It is possible to create an RT } \\
\text { relationship between two concepts (A RT } \\
\text { B). The tool does not insert the } \\
\text { reciprocal relationship). } \\
\text { NM: It is not possible to state that two } \\
\text { concepts are related by an RT } \\
\text { relationship. }\end{array}$ \\
\hline 14 & Create a Top Term & $\begin{array}{l}\text { Create a Top } \\
\text { Concept }\end{array}$ & $\begin{array}{l}\text { M+I: It is possible to state that a Concept } \\
\text { is a Top Concept of another concept } \\
\text { (isTopConceptOf property). The tool } \\
\text { inserts the reciprocal relationship } \\
\text { (hasTopConcept). } \\
\text { M-I: It is possible to state that a Concept } \\
\text { is a Top Concept of another concept } \\
\text { (isTopConceptOf property). The tool } \\
\text { inserts the reciprocal relationship } \\
\text { (hasTopConcept). } \\
\text { NM: Top Concepts are not supported. }\end{array}$ \\
\hline 15 & & $\begin{array}{l}\text { Insert a Concept in } \\
\text { an Array }\end{array}$ & $\begin{array}{l}\mathrm{M}+\mathrm{I} \text { : It is possible to state that a Concept } \\
\text { is a member of some Array } \\
\text { (isMemberOfArray property). The tool } \\
\text { inserts the reciprocal relationship } \\
\text { (hasMemberConcept). } \\
\text { M-I: It is possible to state that a Concept } \\
\text { is a member of some Array } \\
\text { (isMemberOfArray property). The tool }\end{array}$ \\
\hline
\end{tabular}




\begin{tabular}{|l|l|l|}
\hline & & $\begin{array}{l}\text { does not insert the reciprocal relationship } \\
\text { (hasMemberConcept). } \\
\text { NM: It is not possible to insert Concepts } \\
\text { in Arrays. }\end{array}$ \\
\hline
\end{tabular}

